\title{
Thermoelectrically Coupled Nanoantennas for Solar Research
}

\author{
Gary H. Bernstein ${ }^{1}$, David Burghoff ${ }^{1}$, David G. Gonzalez ${ }^{1}$, Edward C. Kinzel², Alexei O. Orlov ${ }^{1}$, \\ Wolfgang Porod $^{1}$, David Strobel ${ }^{3}$, Gergo P. Szakmany ${ }^{1}$, and Stephen M. White ${ }^{4}$ \\ ${ }^{1}$ Department of Electrical Engineering, University of Notre Dame \\ 275 Fitzpatrick Hall, Notre Dame, IN 46556, USA \\ gbernste@nd.edu; dgarci23@nd.edu; dburghoff@nd.edu; aorlov@nd.edu; porod@nd.edu; gszakman@nd.edu \\ ${ }^{2}$ Department of Aerospace and Mechanical Engineering, University of Notre Dame \\ 365 Fitzpatrick Hall, Notre Dame, IN 46556, USA \\ ekinzel@nd.edu \\ ${ }^{3}$ Space Micro Inc. \\ 15378 Avenue of Science, San Diego, CA 92128, USA \\ dstrobel@spacemicro.com \\ ${ }^{4}$ Space Vehicles Directorate \\ 1006 Bernalillo Pl SE \\ Albuquerque NM 87123 \\ stephen.white.24@us.af.mil
}

\begin{abstract}
We are developing thermoelectrically coupled nanoantennas (TECNAs) as infrared (IR) sensors for applications in solar imaging in the mid- and far-IR, both terrestrially and for future space missions. Nanoantennas are nanoscale structures that interact with light in the same way that macroscale antennas interact with radio waves. The electric field of the electromagnetic wave causes currents in the antenna, which interact with an amplifier to produce a signal. In our nanoantennas, the electric field of the IR radiation induces currents that heat the nanowire antenna and, subsequently, a nanothermocouple that provides the signal to our amplifier. IR sensors exhibit various characteristics that include wavelength selectivity, sensitivity (specific detectivity, D*, or noise equivalent temperature difference, NETD), speed (cadence), polarization sensitivity, operating temperature, directionality, power consumption, and cost of manufacture. Various commercial IR sensors excel in one or only a few of these characteristics. Our uncooled IR detectors can be made sensitive from a few microns to sub-THz, and compete very favorably in all of these characteristics, except for sensitivity, in a single general design. This paper covers the motivation for developing TECNAs for solar imaging, nanothermocouples, including a 'monometallic nanothermocouple' developed at Notre Dame, thermal and electrical properties of our nanoantennas, fabrication, performance of cadence into the $100 \mathrm{~s}$ of $\mathrm{kHz}$, and polarization sensitivity.
\end{abstract}

Keywords: long-wave IR, Nanoantenna, Seebeck effect, Solar imaging, Thermocouple, Thermoelectricity

\section{Introduction}

Solar radiation in the wavelength range between $3 \mu \mathrm{m}$ and $100 \mu \mathrm{m}$ is rarely studied, but is of interest in order to better understand solar flare activity and related physics. Recent studies of flare emission at $\lambda=10 \mu \mathrm{m}$ show similarities to the white-light emission, rather than to the expected radio source observations [1]. Therefore, further studies of the Sun in the $\mathrm{mid} / \mathrm{far}$ IR are required. However, terrestrial-based observation of the Sun in the mid-wave infrared regime (MWIR) between $5 \mu \mathrm{m}$ to about $20 \mu \mathrm{m}$ is hard to perform due to atmospheric absorption, and is completely obscured beyond about $30 \mu \mathrm{m}$. A new type of detector is required for this spectral range that provides multi-spectral and polarization sensitive capabilities. Also, possible space-based observations in the long-wave IR regime (LWIR) out to $100 \mu \mathrm{m}$ will provide additional data to be used in solar emission models.

MWIR and LWIR detectors have significantly advanced over the last couple of decades, and their operation is commonly based on the conversion of photons to either charge carriers or thermal energy. Photovoltaic devices are based on the absorption of photon energy that changes the charge distribution inside the detector material, resulting in an electrical signal that is proportional to the incident radiation [2]. The photon energy between $5 \mu \mathrm{m}$ to $20 \mu \mathrm{m}$ is between 0.3 
$\mathrm{eV}$ and $0.06 \mathrm{eV}$. As a result, significant bandgap engineering, and external cooling, is required to overcome the thermal noise [3]. Thermal-based detectors, most commonly microbolometers, absorb the incident IR radiation as heat. This heating effect causes a measurable physical property change, usually in the resistance, that is proportional to the intensity of the incident radiation [2]. A third, and less common, class of LWIR detectors is the radiation-field type that exploits the wave nature of the IR radiation, as reported here. These devices employ a resonant structure that transduces the optical energy to electrical currents that are rectified through heating of a thermocouple.

Commercial IR sensors possess various characteristics including spectral selectivity, sensitivity, speed, polarization sensitivity, operating temperature, directionality, power consumption, and cost of manufacture. Any given sensor is superior in only one or a few of these properties. In this paper, we present our uncooled thermoelectrically coupled nanoantennas (TECNA) that are a radiation-field type IR detector. They compete vary favorably in all of these characteristics, except as yet for sensitivity, in one general design. TECNAs are constructed from a dipole antenna and a nanothermocouple (NTC), as shown in Fig. 1a. The incident IR radiation is resonantly absorbed by the nanoantenna, and the radiation-induced antenna currents heat the hot junction of a nanothermocouple by Joule heating. The nanothermocouple converts the heat to an electrical signal by the Seebeck effect. These devices are uncooled [4-6], frequency selective [7], polarization sensitive [8], fast [9] and operate without an external power supply [4]. They can be tailored to be sensitive from a few microns to sub-THz [7], a range of up to two orders of magnitude.
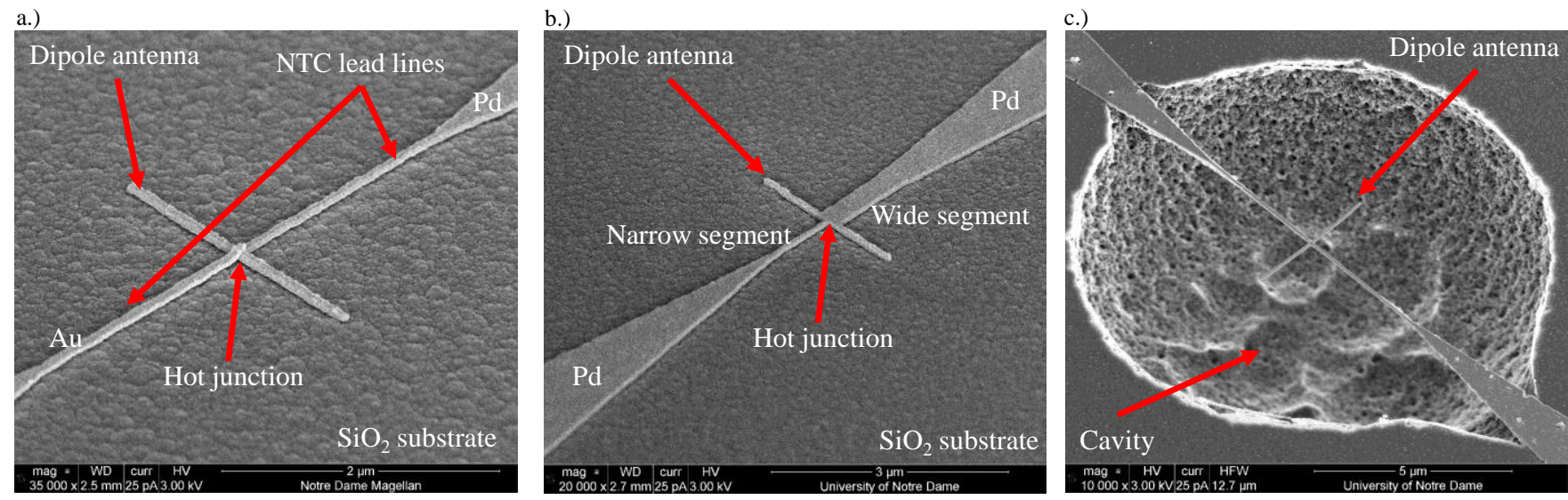

Fig. 1: SEM micrographs of TECNAs. (a) A bi-metal TECNA on a $\mathrm{SiO}_{2}$ substrate. The NTC is based on traditional thermocouples, i.e., the two conductors are from dissimilar metals. (b) A single-metal TECNA on a $\mathrm{SiO}_{2}$ substrate. The NTC is formed from a single layer of Pd where the non-zero relative Seebeck coefficient is the result of the unequal nanowire segment widths. (c) Suspended TECNA above a cavity etched into the Si substrate.

Nanothermocouples can be constructed, as are macroscale thermocouples, from two different metallic nanowires. Their open-circuit voltage response, $V_{O C}$, to a temperature gradient, $\Delta T$, between their hot and cold junctions can be expressed as

$$
V_{O C}=\Delta \mathrm{T} \times \Delta \mathrm{S}
$$

where $\Delta S$ is the relative Seebeck coefficient (RSC) of the NTC. However, at the nanoscale, thermocouples can be constructed from a single layer of metal with shape engineering as introduced by us [10]. At the nanoscale, the charge transport properties of metals, including the absolute Seebeck coefficient, are size-dependent and differ from their thin-film and bulk values due to increased electron scattering at the surface and grain boundaries [11, 12]. As a result, a non-zero relative Seebeck coefficient for NTCs can be achieved by constructing them from a single layer of film with two different cross sections. The hot junction of such NTCs is formed at the cross-sectional discontinuity, where the nanowire changes dimension, as shown in Fig. 1b. 
Conventional bolometer-based IR detectors comprise a large thermal mass that can heat and cool only slowly, with frequencies up to a few hundred Hz. Because TECNAs are based on nanoantennas that resonantly absorb the incident IR radiation using a dipole antenna, their response time is much faster than that of thermal detectors. The thermal mass of a dipole antenna and lead lines is on the order of a few tens of $\mathrm{fJ} / \mathrm{K}$, which is extremely small compared to microbolometers, and thus enables operation in the $\mathrm{MHz}$ regime [13].

\section{Design \& Fabrication}

\subsection{Substrate Effect}

As Eq. 1 shows, the thermal response of the TECNAs depends on both the RSC and the antenna-produced differential

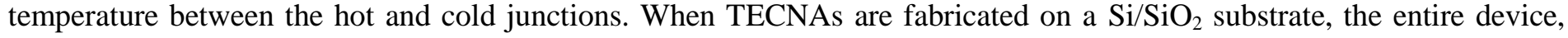
including the antenna and the hot junction, are in thermal contact with the substrate. Figure 2a compares the temperature distribution along the antenna on a substrate and in air demonstrating that contact with the substrate removes the antennaproduced heat very effectively. Consequently, thermal insulation of TECNAs from the substrate is necessary to increase their sensitivity. We have shown that by etching a spherical cavity in the Si substrate under the antenna, as shown in Fig. 1c, the device response increases by about 100 times [14]. Besides thermal insulation, the cavity also acts as an optical element and focuses the incident IR radiation toward the antenna $[14,15]$. For a spherical cavity, and within the paraxial approximation model, the focal point is $f=D / 4$, where $D$ is the aperture size of the cavity.

\subsection{Antenna Design}

Nanoantennas are resonant structures that selectively couple to those wavelengths that are matched to their physical lengths. We use COMSOL Multiphysics simulations to determine the resonant antenna length, which at the nanoscale depends on the antenna material properties [16] and the dielectric constant of the media that surround it [17]. In the simulations, the antennas are excited at their center using a lumped port, and the antenna length is varied. The antenna is at resonance when the reflection coefficient, $S_{1 l}$, is a minimum. Figure $2 \mathrm{~b}$ shows the reflection coefficient as a function of antenna length for $\mathrm{Au}, \mathrm{Cr}, \mathrm{Cu}, \mathrm{Ni}$, and $\mathrm{Pd}$ dipole antennas designed for $10.6 \mu \mathrm{m}$ radiation. For conventional macroantennas, the radiation-induced current flows mainly on the surface due to the skin effect. However, the thickness of our nanoantennas is on the order of the skin depth at LWIR frequencies, from about $10 \mathrm{~nm}$ to $40 \mathrm{~nm}$ [18], so the nanoantenna currents propagate inside the antenna. Thus, the resonant length is material dependent, as shown in Fig. $2 \mathrm{~b}$. The electrical properties of the metals at $10.6 \mu \mathrm{m}$ are obtained from $[19,20]$. The calculated port impedance also indicates resonance when the reactive component is zero. In this case, the calculated antenna impedance is purely resistive, $R \sim 82 \Omega$, as shown in Fig. 2c. Figure $2 \mathrm{~b}$ also shows that the bandwidth of the nanoantennas is about $\pm 1 \mu \mathrm{m}$ around the center wavelength.
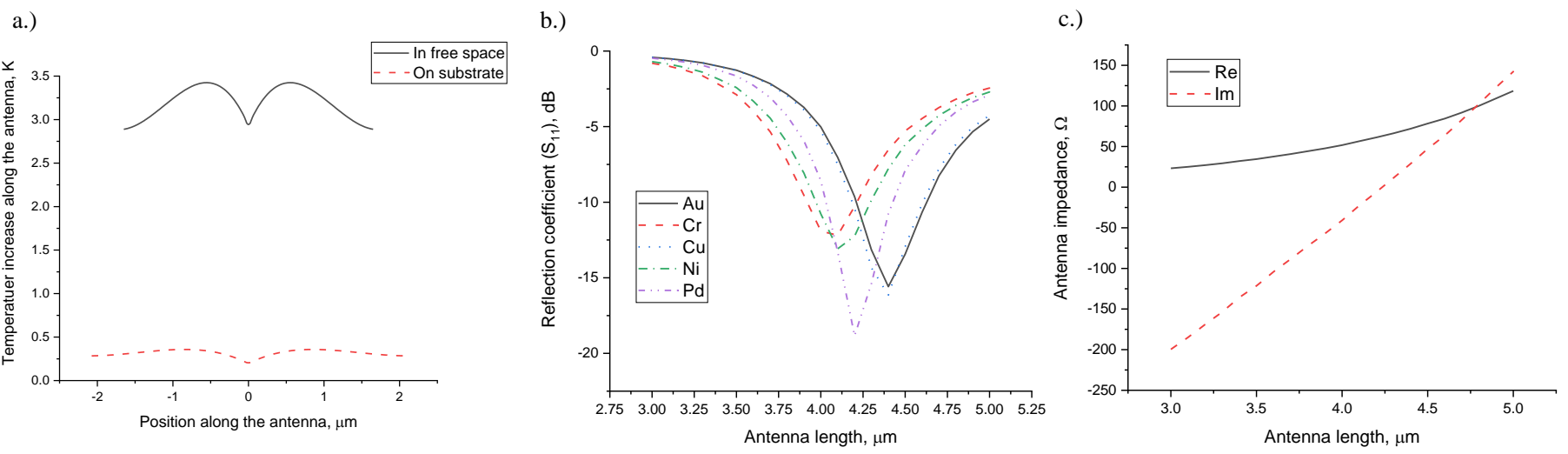

Fig. 2: COMSOL simulations of TECNAs. (a) Temperature profiles along an antenna with attached lead lines both in air and on a silicon substrate show that the substrate very effectively removes the antenna-produced heat, thus reducing its sensitivity. (b)The resonant antenna length is determined by minimizing the reflection coefficient. (c) Simulated Pd antenna impedance as a function of antenna length for $10.6 \mu \mathrm{m}$ excitation. When the reactive (imaginary) component is zero, the antenna is at resonance. 


\subsection{Fabrication}

The fabrication of the suspended TECNAs involves optical and electron beam lithography, thin-film deposition and etching. First, bonding pads and lead lines that are used for electrical and optical measurements are patterned and formed from a $200 \mathrm{~nm}$ thick Au layer on top of a high-resistivity Si wafer. Then, the TECNAs and lead lines are exposed into a PMGI/PMMA double-layer resist structure by a Raith EBPG 5200 electron beam lithography system. Then the antenna and the NTC lead lines are e-beam evaporated and lifted off. Finally, a circular etch window is exposed around the nanoantenna, and the $\mathrm{Si}$ substrate is etched by $\mathrm{XeF}_{2}$ to form the cavity under the antenna. The interested reader will find the fabrication details in [21].

\section{Measurement}

A $\mathrm{CO}_{2}$ laser, $\lambda=10.6 \mu \mathrm{m}$, emitting $1.4 \mathrm{~W} / \mathrm{cm}^{2}$ is used as an IR source for optical testing of the TECNAs. The output of the laser is passed through a linear polarizer, and a half-wave plate is used to set the polarization angle to be parallel to the antenna's long axis. The laser beam is square-wave modulated by an acousto-optic modulator. The $V_{O C}$ response is measured by a custom-made differential voltage amplifier and lock-in technique.

We have connected up to several hundred nanoantennas in series to create a thermopile that provides a relatively large VOC. The polarization-dependent response of a thermopile is shown in Fig. 3a. When the polarization of the incident laser beam is parallel to the antennas, maximum $V_{O C}$ is measured, and when the polarization is perpendicular, minimum response is measured. The polarization-dependent response follows the cosine-square dependence expected for a dipole antenna from classical antenna theory [22]. Therefore, it confirms that the antennas resonantly absorb the incident IR radiation that heats the hot junction of the nanothermocouple. In particular, Fig. 3a is the polarizationdependent response of a thermopile constructed from up to 200 TECNAs connected in series, as shown in Fig 3b. As a result, the SNR of the sensor is increased by $\sqrt{N}$, where $\mathrm{N}$ is the number of TECNAs in the thermopile. Figure $3 \mathrm{c}$ shows that the thermopiles follow the thermocouple addition rule, i.e., as the number of TECNAs in the thermopiles linearly increases the $V_{O C}$ response also linearly increases. These data demonstrate that fabrication of large suspended TECNA thermopiles is possible.

a.)

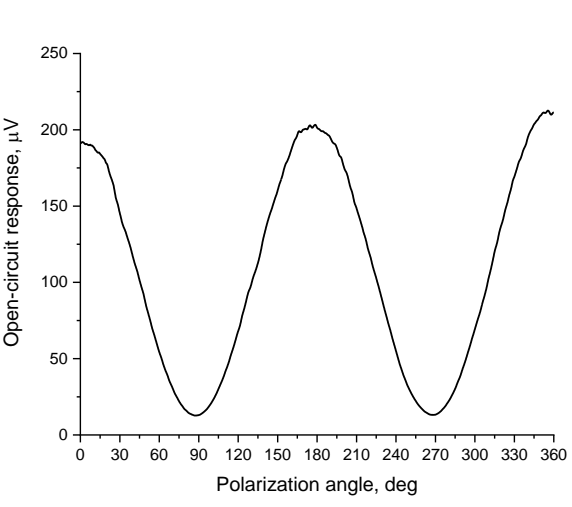

b.)

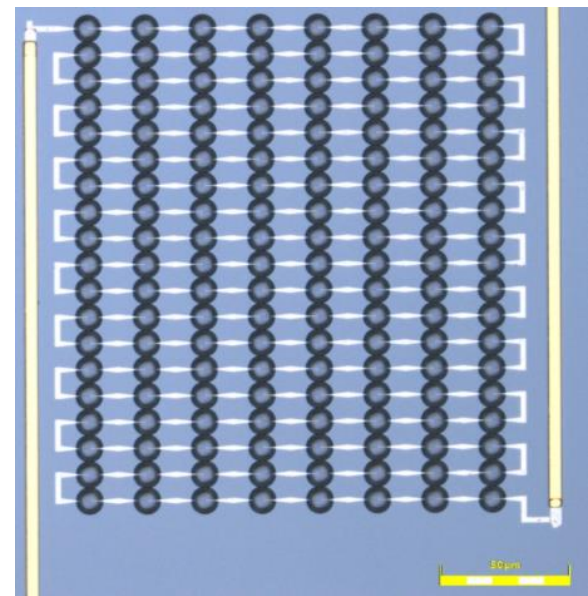

c.)

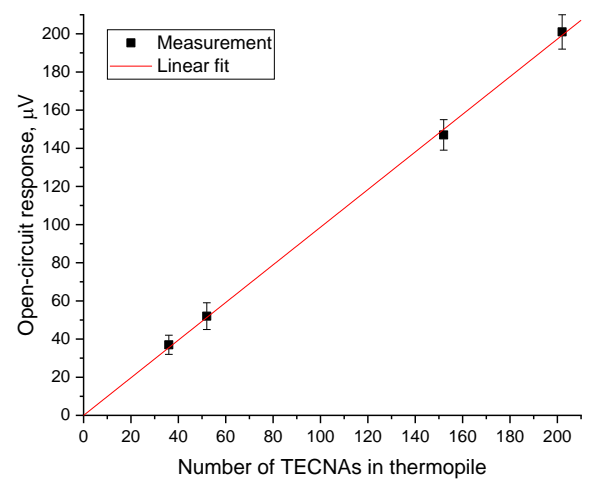

Fig. 3: (a) Polarization-dependent response of a thermopile constructed from 200 TECNAs connected in series. (b) Optical micrograph of the thermopile. (c) The thermopile response linearly increases as the number of TECNAs in the thermopile increases.

We calculate that our specific detectivities, $D^{*}$, are about $5 \times 10^{7}$ Jones, which is about half an order of magnitude less sensitive than commercial bolometer-based mid-IR sensors. High sensitivity is generally required for focal plane arrays used in imaging applications. Irradiance from the Sun's blackbody radiation at air mass 1 (at sea level) 
integrated from 9.6 to $11.6 \mu \mathrm{m}$ (the bandwidth of the TECNA) is about $0.5 \mathrm{~W} / \mathrm{m}^{2}$. This drops off by a few orders of magnitude at about $100 \mu \mathrm{m}$ (at air mass 0, i.e., exoatmospheric), so improving detectivity is an important goal. A telescope telescope with a diameter of $0.6 \mathrm{~m}$ and $\mathrm{f} / \#=4$ will increase the power density at the focal plane by about 330 times. Even Even with the telescope, the irradiance around $10.6 \mu \mathrm{m}$ will be about 100 times less than that of our laser. We have already already demonstrated that we can detect modulation of the laser at frequencies up to $1 \mathrm{MHz}$ [13], so by integrating or averaging our signal for relatively long times, we should be able to capture signals at up to 1000 frames per second, although these speeds may not be necessary for the specific application of solar imaging. Longer wavelengths will be more challenging because the irradiance is much lower, but we can add more thermocouples to the larger antennas and capture more of the thermal energy, increasing $\mathrm{D}^{*}$.

\section{Conclusion}

In this paper, we have shown that TECNAs are uncooled, radiation-field type mid- and long-wave IR detectors that are also polarization sensitive and can form large arrays for imaging applications. The antenna geometry determines the spectral range of the devices with about a $2 \mu \mathrm{m}$ bandwidth for IR sensing. In addition, the antenna also provides polarization sensitivity and directionality. When the devices are thermally insulated from a substrate by suspending them over a cavity etched into the Si substrate, their response increases about 100 times compared to uninsulated devices.

Although, the current TECNA design allows the development of a multi-spectral, polarization sensitive, fast FPA operating in the MWIR and LWIR regimes to image the Sun, the sensitivity of these devices is not yet competitive compared to commercial detectors. However, by increasing the reflectance of the cavity and using thermoelectrically moreefficient materials for the NTCs rather than metals, the specific detectivity could potentially increase to the $10^{9} \mathrm{Jones}$ range. This is competitive with microbolometers, but with added benefits such as polarization sensitivity, wavelength selectivity to the $\mathrm{THz}$, and operation without an external power source.

\section{Acknowledgements}

This work was supported by NASA under grant\# 80NSSC20K0918 and NSF under grant\# NSF CMMI 1947391. The material contained in this document is based upon work supported by a National Aeronautics and Space Administration (NASA) grant or cooperative agreement. Any opinions, findings, conclusions or recommendations expressed in this material are those of the authors and do not necessarily reflect the views of NASA.

\section{References}

[1] P. Kaufmann, S. M. White, S. L. Freeland, R. Marcon, L. O. T. Fernandes, A. S. Kudaka, R. V. de Souza, J. L. Aballay, G. Fernandez, R. Godoy, A. Marun, A. Valio, J. P. Raulin, and C. G. Giménez de Castro, "A Bright Impulsive Solar Burst Detected at 30 THz," The Astrophysical Journal, vol. 768, p. 134, 2013.

[2] G. D. Boreman and E. L. Dereniak, Infrared detectors and systems: New York : Wiley, 1996.

[3] A. Rogalski, Next decade in infrared detectors vol. 10433: SPIE, 2017.

[4] G. P. Szakmany, P. M. Krenz, A. O. Orlov, G. H. Bernstein, and W. Porod, "Antenna-Coupled Nanowire Thermocouples for Infrared Detection," IEEE Trans. Nanotechnol., vol. 12, pp. 163-167, 2013.

[5] E. Briones, A. Cuadrado, J. Briones, R. Díaz de León, J. C. Martínez-Antón, S. McMurtry, M. Hehn, F. Montaigne, J. Alda, and F. J. González, "Seebeck Nanoantennas for the Detection and Characterization of Infrared Radiation," Opt. Express, vol. 22, pp. A1538-A1546, 2014.

[6] H. M. Mubarak, O. Sidek, R. M. Abdel-Rahman, T. M. Mustaffa, S. A. Mustapa Kamal, and M. S. Mukras, "Nano-Antenna Coupled Infrared Detector Design," Sensors, vol. 18, 2018.

[7] G. P. Szakmany, A. O. Orlov, G. H. Bernstein, and W. Porod, "THz Wave Detection by Antenna-Coupled Nanoscale Thermoelectric Converters," IEEE Trans. Terahertz Sci. Technol., vol. 7, pp. 582 - 585, 2017.

[8] G. P. Szakmany, A. O. Orlov, G. H. Bernstein, and W. Porod, "Polarization-Dependent Response of Single- and Bi-Metal Antenna-Coupled Thermopiles for Infrared Detection," IEEE Trans. Terahertz Sci. Technol., vol. 5, pp. 884-891, 2015. 
[9] G. P. Szakmany, A. O. Orlov, G. H. Bernstein, and W. Porod, "Evaluating the Frequency Response of Nanoscale Thermocouples Using Temperature Oscillations in Nanoscale Heaters," IEEE Transactions on Nanotechnology, vol. 15, pp. 567-573, 2016.

[10] G. P. Szakmany, A. O. Orlov, G. H. Bernstein, and W. Porod, "Single-Metal Nanoscale Thermocouples," IEEE Trans. Nanotechnol., vol. 13, pp. 1234-1239, 2014.

[11] D. C. Chu, D. T. Bilir, R. F. W. Pease, and K. E. Goodson, Thin film nano thermocouple sensors for applications in laser and electron beam irradiation. New York: IEEE, 2003.

[12] C. R. Tellier, "A theoretical description of grain boundary electron scattering by an effective mean free path," Thin Solid Films, vol. 51, pp. 311-317, 1978.

[13] G. P. Szakmany, G. H. Bernstein, E. C. Kinzel, A. O. Orlov, and W. Porod, "High Speed Nanoantenna Thermopiles for Long-Wave Infrared Detection," in 2020 IEEE Silicon Nanoelectronics Workshop (SNW), 2020, pp. 113-114.

[14] G. P. Szakmany, A. O. Orlov, G. H. Bernstein, and W. Porod, "Cavity-Backed Antenna-Coupled Nanothermocouples," Sci. Rep., vol. 9, p. 9606, 2019.

[15] M. H. Mubarak, O. Sidek, M. R. Abdel-Rahman, M. T. Mustaffa, A. S. M. Kamal, and S. M. Mukras, "Fabrication of microparabolic reflector for infrared antenna coupled detectors," Micro \& Nano Letters, vol. 13, pp. 1343-1348, 2018.

[16] B. Mora-Ventura, R. D. d. León, G. García-Torales, J. L. F. Nuñez, J. Alda, and F. J. González, "Responsivity and Resonant Properties of Dipole, Bowtie, and Spiral Seebeck Nanoantennas," J. Photonics Energy, vol. 6, p. 8, 2016.

[17] L. Novotny, "Effective Wavelength Scaling for Optical Antennas," Physical Review Letters, vol. 98, p. 266802, 2007.

[18] G. W. Bryant, F. J. García de Abajo, and J. Aizpurua, "Mapping the Plasmon Resonances of Metallic Nanoantennas," Nano Lett., vol. 8, pp. 631-636, 2008.

[19] M. A. Ordal, R. J. Bell, R. W. Alexander, L. L. Long, and M. R. Querry, "Optical properties of fourteen metals in the infrared and far infrared: Al, $\mathrm{Co}, \mathrm{Cu}, \mathrm{Au}, \mathrm{Fe}, \mathrm{Pb}, \mathrm{Mo}, \mathrm{Ni}, \mathrm{Pd}, \mathrm{Pt}, \mathrm{Ag}, \mathrm{Ti}, \mathrm{V}$, and W," Applied Optics, vol. 24, pp. 4493-4499, 1985.

[20] A. D. Rakić, A. B. Djurišić, J. M. Elazar, and M. L. Majewski, "Optical properties of metallic films for verticalcavity optoelectronic devices," Applied Optics, vol. 37, pp. 5271-5283, 1998.

[21] G. P. Szakmany, A. O. Orlov, G. H. Bernstein, and W. Porod, "Fabrication of suspended antenna-coupled nanothermocouples," J. Vac. Sci. Technol., B, vol. 37, p. 052201, 2019.

[22] C. A. Balanis, Antenna Theory: Analysis and Design, 3rd ed. Hoboken, NJ: John Wiley \& Sons, Inc., 2005. 